\title{
9th Grade Completion
}

National Cancer Institute

\section{Source}

National Cancer Institute. 9th Grade Completion. NCI Thesaurus. Code C67131.

Indicates that 9th grade is the highest level of educational achievement. 